\title{
Sirt3 is a tumor suppressor in lung adenocarcinoma cells
}

\author{
KUI XIAO ${ }^{1}$, JIEHAN JIANG $^{2}$, WEI WANG ${ }^{3}$, SHAN CAO $^{4}$, LIMING ZHU $^{5}$, HUIHUI ZENG $^{1}$, \\ RUOYUN OUYANG ${ }^{1}$, RUI ZHOU ${ }^{1}$ and PING CHEN ${ }^{1}$
}

\begin{abstract}
${ }^{1}$ Department of Respiratory Medicine, The Second Xiangya Hospital, Institute of Respiratory Disease, Central South University, Changsha, Hunan 410011; ${ }^{2}$ Department of Respiratory Medicine, Changsha Central Hospital, Changsha, Hunan 410004;

${ }^{3}$ Department of Vascular Surgery, Xiangya Hospital, Central South University, Changsha, Hunan 410008;

${ }^{4}$ Institute of Clinical Pharmacology, Hunan Key Laboratory of Pharmacogenetics, Central South University, Changsha, Hunan 410078; ${ }^{5}$ Department of Respiratory Medicine, Hunan Geriatric Hospital, Changsha, Hunan 410016, P.R. China
\end{abstract}

Received March 28, 2013; Accepted June 18, 2013

DOI: 10.3892/or.2013.2604

\begin{abstract}
Sirt3, a member of the mammalian sirtuin family protein that is localized to mitochondria, is a $\mathrm{NAD}^{+}$-dependent deacetylase and plays an important role in the control of metabolic activity. Recently, several studies have shown the potential role of Sirt3 in certain types of tumors such as breast cancer and hepatocellular carcinoma. However, the role of Sirt3 in lung adenocarcinoma has never been studied. In the present study, we found that Sirt3 protein expression was downregulated in human lung adenocarcinoma tissue when compared with that in adjacent normal tissue. Overexpression of Sirt3 using adenovirus significantly inhibited the growth of the A549 lung adenocarcinoma cell line. In this cell line, overexpression of Sirt3 induced apoptosis, which was evidenced by Annexin V + PI assay and cleaved caspase-3 immunoblotting. Furthermore, overexpression of Sirt3 increased the bax/ bcl-2 and bad/bcl-x/L ratios, and promoted AIF translocation to the nucleus. Finally, Sirt3 overexpression upregulated p53 and $\mathrm{p} 21$ protein levels, and decreased intracellular ROS levels. Collectively, our data suggest that Sirt3 is a tumor suppressor in lung adenocarcinoma development and progression and may be a promising therapeutic target for lung adenocarcinoma.
\end{abstract}

\section{Introduction}

Worldwide, lung cancer is the most common cancer with high incidence and mortality rates $(1,2)$. Non-small cell lung cancer accounts for nearly $80 \%$ of the disease, while lung adenocarcinoma is the most common type of non-small cell

Correspondence to: Professor Ping Chen or Professor Rui Zhou, Department of Respiratory Medicine, The Second Xiangya Hospital, Institute of Respiratory Disease, Central South University, Changsha, Hunan 410011, P.R. China

E-mail: pingchen0731@sina.com

E-mail: zhourui2355@sina.com

Key words: Sirt3, lung adenocarcinoma, apoptosis, reactive oxygen species lung cancer (1). Lung adenocarcinoma is characterized by peripheral location in the lung and often has activating mutations in the $K$-ras oncogene $(3,4)$. Recently, several studies have highlighted the importance of mutations of the epidermal growth factor receptor (EGFR) and other oncogenes $(5,6)$; however, the causes and biology of lung adenocarcinoma are not yet fully understood $(7,8)$.

In cancer cells, uncontrolled cell proliferation involves not only dysfunctions in the control of cell growth and division but also adjustments in energy metabolism that is utilized to support cancer cell proliferation. Several important mutant oncogenes (e.g., TP53) have been reported to be associated with abnormal glycolytic fueling $(9,10)$. Recently, deregulated cellular energy metabolism has been viewed as a new hallmark of cancer (11). As an indispensable cell energy factory for the survival of cells, the mitochondrion is an important regulator of vital and lethal functions, particularly the intrinsic pathway of apoptosis (12). More and more data demonstrate that the mitochondrion is a critical target for cancer therapy $(13,14)$.

Sirtuins are a conserved family of deacetylases and monoADP-ribosyltransferases that use $\mathrm{NAD}^{+}$as a co-substrate (15). There are 7 members of the sirtuin family in mammals (Sirt1-Sirt7) (15). These proteins are believed to be involved in stress response, metabolism and longevity (15). Sirtuins are widely expressed in different tissues and are localized in different subcellular compartments $(15,16)$. Among the 7 members, Sirt3, Sirt4 and Sirt5 are localized in the mitochondrion. Sirt3 is believed to be the major deacetylase within the mitochondrion, since the deacetylase activity of Sirt 4 and Sirt5 is rather weak (17).

The role of Sirt3 in tumor biology has become a topic of increased interest in recent years. Ashraf et al (18) first reported that increased levels of Sirt3 and Sirt7 transcription are associated with node-positive breast cancer. Kim et al (19) demonstrated that gene deletion of Sirt3 facilitates the development of breast tumors in mice, suggesting the tumorsuppressive effect of Sirt3 in breast cancer, which was confirmed by a later study (20). A recent report showed that Sirt3 inhibits hepatocellular carcinoma cell growth through reducing Mdm2-mediated p53 degradation (21). However, the exact role of Sirt3 in other tumors is still being discovered. In 
the present study, we investigated the expression of Sirt3 in human lung adenocarcinoma tissues and explored the potential role of Sirt3 in lung adenocarcinoma.

\section{Materials and methods}

Reagents. Antibodies against Sirt3 and apoptosis-inducing factor (AIF) were purchased from Millipore Chemicon International (Temecula, CA, USA). Antibodies against cleaved caspase-3, bax, bcl-2, bad, bcl-x/L, p53 and p21 were purchased from Cell Signaling Technology, Inc. (Danvers, MA, USA). Antibody against actin was obtained from SigmaAldrich, (St. Louis, MO, USA). The Annexin V + PI kit was purchased from Promega Corporation (Madison, WI, USA). DAPI and dichlorofluorescein diacetate (DCFH-DA) were purchased from Invitrogen Life Technologies (Carlsbad, CA, USA). Enhanced chemiluminescence and protease/phosphatase inhibitors were purchased from Pierce Biotechnology, Inc. (Rockford, IL, USA).

Human lung adenocarcinoma tissue. Four pairs of lung adenocarcinoma and matched normal adjacent tissue extracts were obtained from Chinese patients who underwent surgical resection for diagnosis and therapy in our hospital. Samples were obtained following informed consent according to an established protocol approved by the Ethics Committee of Central South University.

Cell culture. The A549 lung adenocarcinoma cell line was obtained from ATCC (Manassas, VA, USA). Cells were cultured in Dulbecco's modified Eagle's medium (DMEM) supplemented with $10 \%$ (v/v) fetal bovine serum (FBS) in $95 \%$ $\mathrm{O}_{2}$ and $5 \% \mathrm{CO}_{2}$.

Construction of the adenovirus expressing Sirt3. The adenovirus expressing Sirt3 (Ad-Sirt3) or the control adenovirus expressing GFP (Ad-GFP) were generated using the RAPAd ${ }^{\circledR}$ CMV adenoviral expression system (Cell Biolabs, Inc., San Diego, CA, USA) according to the manufacturer's protocol as previously described (21). Briefly, mouse Sirt3 cDNA was cloned into pacAd5 CMV-IRES vector. Then, pacAd5 CMV-IRES-Sirt3 and pacAd5 9.2-100 backbone vectors were linearized by $P a c \mathrm{I}$. The purified linearized DNAs were cotransfected into 293 cells using Lipofectamine ${ }^{\mathrm{TM}}$ Plus (Invitrogen Life Technologies). On day 8, adenoviruscontaining cells and media were harvested. Viruses were released by 3 freeze/thaw cycles and stored at $-80^{\circ} \mathrm{C}$. For virus transfection, $20 \mu \mathrm{l}$ of viral stock solution was added into the culture medium $(2 \mathrm{ml})$ for $6 \mathrm{~h}$.

Cell viability assay. Cell viability was evaluated by a nonradioactive Cell Counting Kit-8 (CCK-8) assay as described previously $(21,22)$. Ad-GFP- and Ad-Sirt3-transfected cells $\left(5 \times 10^{3}\right)$ were seeded into 48 -well plates and cultured overnight to allow attachment. After being serum-starved for $8 \mathrm{~h}, \mathrm{FBS}$ was added into the medium. At 12, 24, 36 and $48 \mathrm{~h}$, cells were incubated with $10 \mu \mathrm{l}$ of CCK-8 solution for $3 \mathrm{~h}$ at $37^{\circ} \mathrm{C}$, and then the optical density at $450 \mathrm{~nm}$ was analyzed using a microplate reader (Tecan, Switzerland). Experiments were performed in duplicate.
Quantitative real-time PCR analysis. Real-time PCR analysis was performed on an ABI Prism 7500 sequence detection system using PrimerScript ${ }^{\circledR}$ RT reagent kit (Takara Bio, Inc., Shiga, Japan). The total RNA was extracted form human tissue using TRIzol (Invitrogen Life Technologies) and $5 \mu \mathrm{g}$ of RNA as first-strand cDNA was used. cDNA (100 ng) was amplified using primers as follows: Sirt3 sense, ACAGCAACCTCCAG CAGTACGA and antisense, CGTGTAGAGCCGCAGAAG CA; $\beta$-actin sense, GCACTCTTCCAGCCTTCCTTCC and antisense, CCGCCAGACAGCACTGTGTT. The mRNA levels of housekeeping gene $\beta$-actin were used as control.

Immunochemistry. Immunochemistry was performed as described previously $(23,24)$. Briefly, the A549 cells were seeded onto coverslips and transfected with Ad-GFP or Ad-Sirt3 for $6 \mathrm{~h}$. At $24 \mathrm{~h}$ after transfection, cells were washed twice with PBS and then fixed with buffer containing $4 \%$ paraformaldehyde and $0.1 \%$ Triton $\mathrm{X}-100$ at room temperature for $20 \mathrm{~min}$, followed by incubation with an antibody against $\mathrm{AIF}$ at $37^{\circ} \mathrm{C}$ for $2 \mathrm{~h}$ and incubation with Alexa Fluor 555-conjugated secondary antibody. DAPI was used to stain the nucleus.

Immunoblotting. Immunoblotting analyses of cell extracts were performed as described previously $(22,25)$. Human tissues or cells were lysed with RIPA buffer with protease inhibitor/protein phosphatase inhibitors. Samples were subjected to $10 \%$ SDS-PAGE, and transferred onto PVDF membranes at $100 \mathrm{~V}$ for $1-2 \mathrm{~h}$. After being blocked in blocking buffer for $4 \mathrm{~h}$, the membrane was incubated with a specific primary antibody and then followed by the HRP-labeled secondary antibody, and investigation using the enhanced chemiluminescence system (Pierce Biotechnology, Inc., Rockford, IL, USA).

Annexin $V+P I$ staining assay. The Annexin V + PI assay for apoptosis was performed using flow cytometry as described previously (23). Briefly, Ad-GFP- and Ad-Sirt3transfected cells were trypsinized, washed in ice-cold PBS and re-suspended in $1 \mathrm{ml}$ of the supplied buffer $\left(1 \times 10^{6}\right.$ cells/ ml). A $100 \mu \mathrm{l}$ sample ( $1 \times 10^{5}$ cells) was incubated with $5 \mu \mathrm{l}$ FITC-conjugated Annexin V and $5 \mu \mathrm{l}$ PI for $30 \mathrm{~min}$ at $25^{\circ} \mathrm{C}$ in the dark. Then, cells were analyzed immediately using a BD FACSCalibur (BD Biosciences, Franklin Lakes, NJ, USA). The apoptotic cells were estimated as the percentage of cells that stained positive for FITC-Annexin $\mathrm{V}$ while remaining impermeable to PI (Annexin $\mathrm{V}^{+} / \mathrm{PI}^{-}$).

Statistical analysis. Data are expressed as means \pm SEM. Differences were evaluated by a two-tailed Student's t-test or ANOVA followed by Tukey's post hoc test. Statistical significance was set at $\mathrm{P}<0.05$.

\section{Results}

Sirt3 is downregulated in human lung adenocarcinoma tissue. As shown in Fig. 1A, Sirt3 mRNA levels were significantly downregulated ( $50-60 \%)$ in human lung adenocarcinoma tissue when compared with normal adjacent tissue. Similarly, a marked downregulation of Sirt3 protein levels in human lung adenocarcinoma tissue was observed (Fig. 1B). 
A
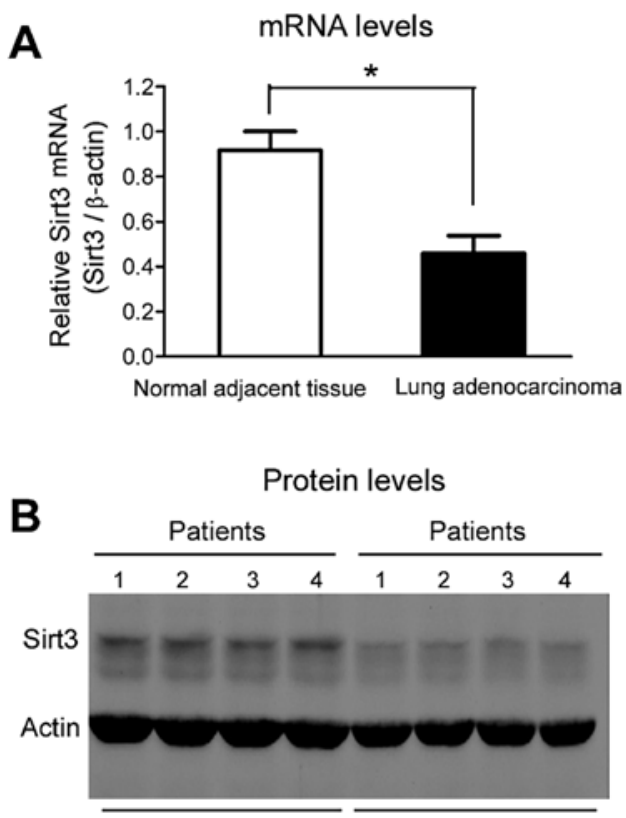

Normal adjacent tissue Lung adenocarcinoma

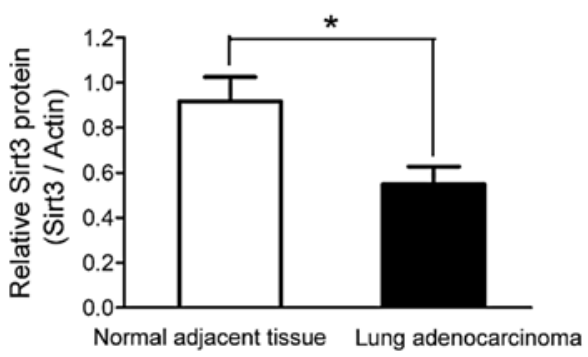

Figure 1. Downregulation of Sirt3 in human lung adenocarcinoma tissue. (A) mRNA level of Sirt3 in human lung adenocarcinoma tissue was measured by real-time PCR analysis. ${ }^{*} \mathrm{P}<0.05$ vs. normal tissue. $\mathrm{n}=4$. (B) Immunoblotting and quantitative analysis of the expression of Sirt3 in human lung adenocarcinoma and normal adjacent tissues. ${ }^{*} \mathrm{P}<0.05$ vs. normal tissue. $n=4$.

Overexpression of Sirt 3 inhibits lung adenocarcinoma cell growth. We next tested whether overexpression of Sirt3 influences A549 lung adenocarcinoma cell growth in vitro. Overexpression of Sirt3 using adenovirus (Fig. 2A) significantly inhibited the growth of A549 cells (Fig. 2B).

Overexpression of Sirt3 induces apoptosis in lung adenocarcinoma cells. We studied the effects of overexpression of Sirt3 on the apoptosis of lung adenocarcinoma cells. First, we analyzed Ad-GFP- and Ad-Sirt3-transfected cells with the FITC-Annexin V + PI staining assay. As shown in Fig. 3A, the ratio of apoptotic cells (Annexin $\mathrm{V}^{+} / \mathrm{PI}^{-}$) was $\sim 0.9 \%$ in the control Ad-GFP-transfected cells, whereas the apoptotic ratio increased to $4.2 \%$ in Ad-Sirt3-transfected cells.

Then, we analyzed the levels of cleaved caspase-3, a key mediator and marker of apoptosis. As shown in Fig. 3B, cleaved caspase-3 expression was detected in the cells overexpressing Sirt3 (Ad-Sirt3-transfected cells) but not in the control cells (Ad-GFP-transfected cells).

Overexpression of Sirt3 modulates apoptotic-related proteins in lung adenocarcinoma cells. The Bcl-2 family proteins and AIF are important determinants regulating cellular apoptosis.
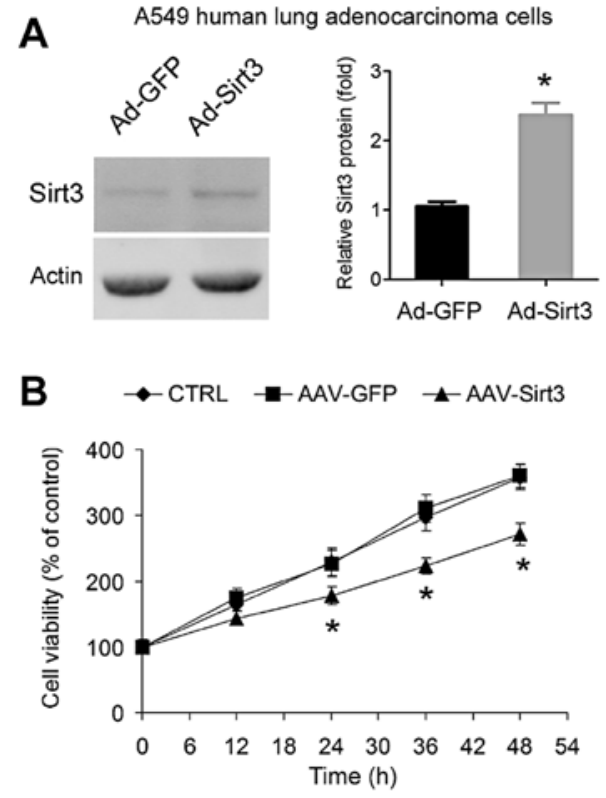

Figure 2. Sirt3 overexpression inhibits lung adenocarcinoma cell growth (A) Immunoblotting demonstrating the overexpression of Sirt3 using adenovirus in A549 lung adenocarcinoma cells. ${ }^{*} \mathrm{P}<0.05$ vs. Ad-GFP (vector control). $n=4$. (B) Cell growth curve of A549 lung adenocarcinoma cells transfected with Ad-GFP or Ad-Sirt3. ${ }^{*} \mathrm{P}<0.05$ vs. Ad-GFP (control). $\mathrm{n}=8$.
A

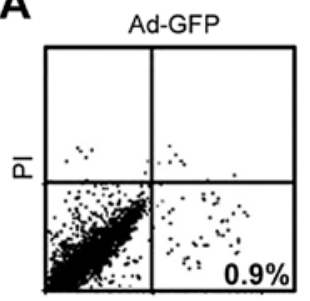

FITC-Annexin V

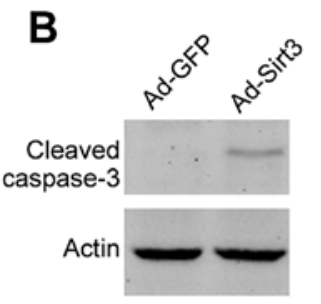

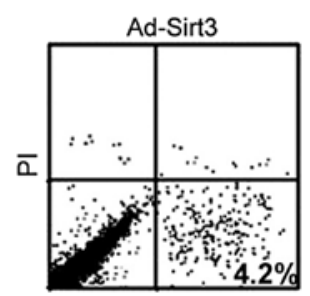

FITC-Annexin V

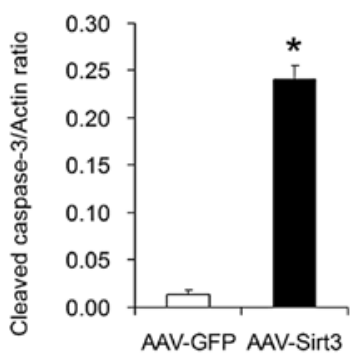

Figure 3. Apoptosis of lung adenocarcinoma cells induced by Sirt3 overexpression. (A) Annexin V + PI assay demonstrating the proportion of apoptotic cells in Sirt3-overexpressing A549 lung adenocarcinoma cells (Ad-Sirt3) and control A549 lung adenocarcinoma cells. (B) Immunoblotting demonstrating cleaved caspase-3 expression in Sirt3-overexpressing and control cells. ${ }^{*} \mathrm{P}<0.05$ vs. Ad-GFP (control). $\mathrm{n}=5$.

Overexpression of Sirt3 increased the bax-bcl-2 ratio and bad-bcl-x/L ratio (Fig. 4A). Moreover, immunofluorescence analysis (Fig. 4B) showed that Sirt3 overexpression induced AIF nuclear translocation.

Overexpression of Sirt 3 upregulates the p53 signaling pathway in lung adenocarcinoma cells. Next, we studied 


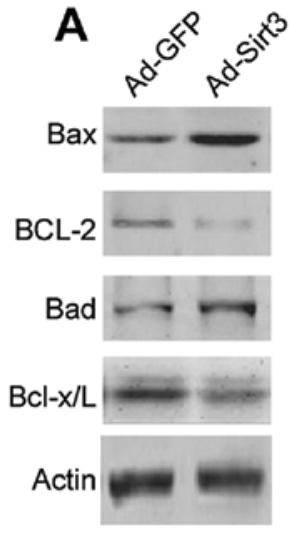

$\mathrm{Bax}$ to $\mathrm{Bcl}-2$ ratio

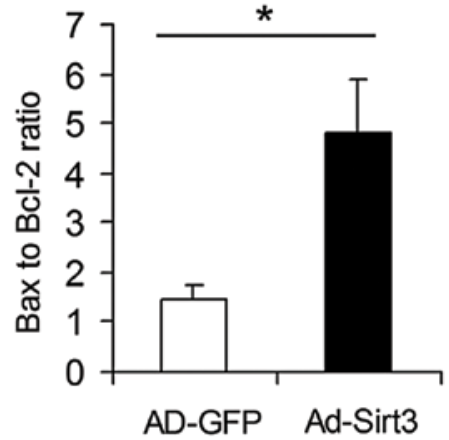

Bad to $\mathrm{Bcl}-\mathrm{x} / \mathrm{L}$ ratio

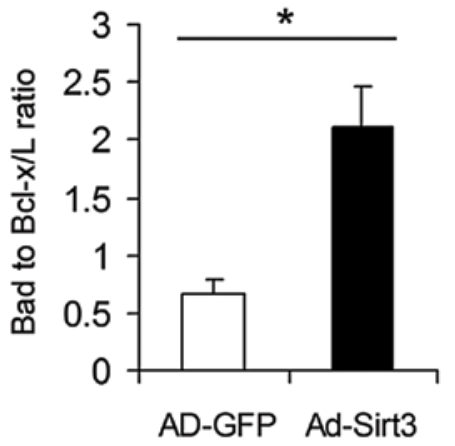

B

AIF

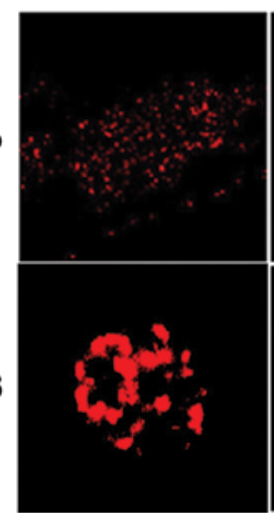

DAPI

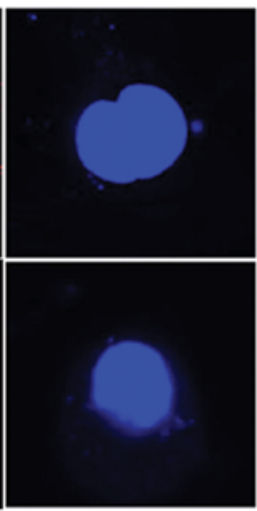

Merge

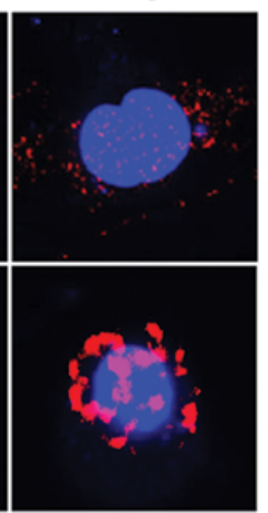

Figure 4. Influence of Sirt3 overexpression on apoptosis-related proteins in lung adenocarcinoma cells. (A) Representative images of immunoblotting and quantitative analysis of bax, bcl-2, bad and bcl-x/L. Actin was used as a loading control. * $\mathrm{P}<0.05$ vs. Ad-GFP. n=6. (B) Representative images of immunofluorescence showing the nuclear translocation of AIF induced by Sirt3 overexpression. AIF was stained by the AIF-specific primary antibody and then the Alexa 555-conjugated secondary antibody. DAPI was used to stain the nucleus.
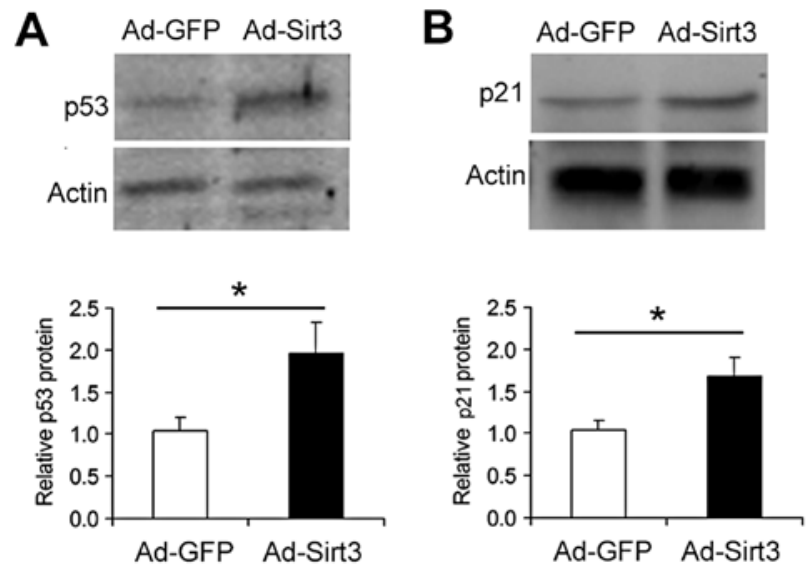

Figure 5. Sirt3 overexpression upregulates p53 and p21 in lung adenocarcinoma cells. (A) Representative images of immunoblotting and quantitative analysis of p53 protein levels. ${ }^{*} \mathrm{P}<0.05$ vs. Ad-GFP. $\mathrm{n}=4$. (B) Representative images of immunoblotting and quantitative analysis of $\mathrm{p} 21$ protein levels. ${ }^{*} \mathrm{P}<0.05$ vs. Ad-GFP. $\mathrm{n}=4$.

the influence of Sirt3 overexpression on p53 signaling in A549 cells. Compared with the Ad-GFP-transfected cells,
Ad-Sirt3-transfected cells exhibited increased protein levels of p53 (Fig. 5A) and p21 (Fig. 5B), a p53-downstream factor.

Overexpression of Sirt3 decreases oxidative stress in lung adenocarcinoma cells. Finally, we studied the influence of Sirt 3 overexpression on oxidative stress in A549 cells. The reactive oxygen species (ROS) generation measured by the DCFH-DA assay (Fig. 6A) revealed that overexpression of Sirt3 significantly decreased the total ROS level in A549 cells (Fig. 6B). Moreover, overexpression of Sirt3 attenuated the superoxide anion level in A549 cells (Fig. 6B).

\section{Discussion}

In the present study, we initially demonstrated that Sirt3 was markedly downregulated in lung adenocarcinoma tissue when compared with that in the normal adjacent tissue. Using adenovirus-mediated overexpression, we found that Sirt3 overexpression inhibited the growth of A549 lung adenocarcinoma cells. Further analyses, including Annexin V + PI assay, cleaved caspase-3 immunoblotting, bax-bcl-2 ratio, bad-bcl-x/L ratio and AIF translocation, showed that Sirt3 overexpression promoted apoptosis in A549 lung adenocarcinoma cells. 

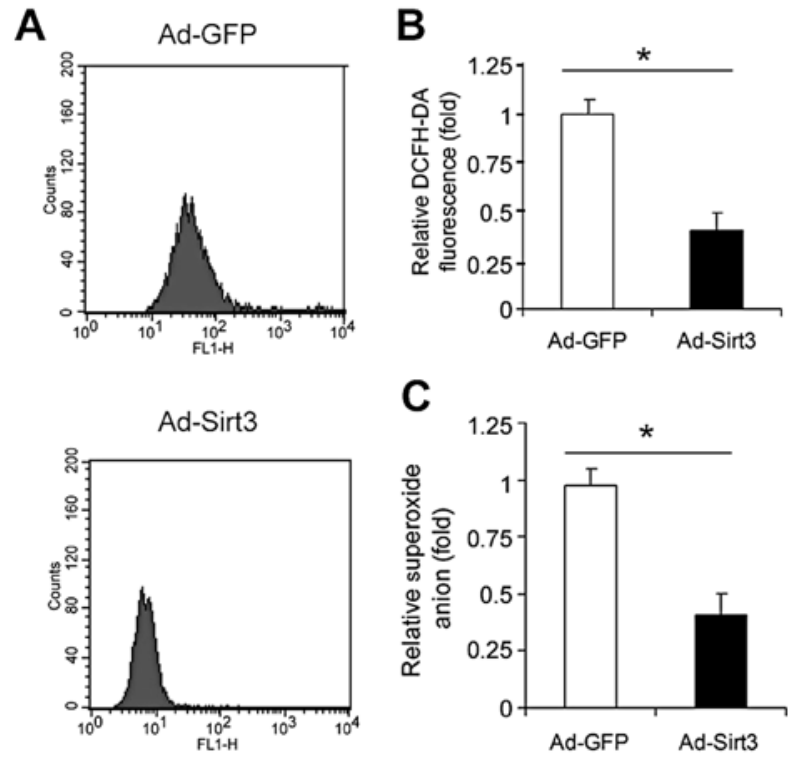

Figure 6. Sirt3 overexpression decreases the ROS level in lung adenocarcinoma cells. (A and B) Dichlorofluorescein diacetate (DCFH-DA) fluorescent intensity was assessed by flow cytometric analysis and shown in the histogram. ${ }^{*} \mathrm{P}<0.05$ vs. Ad-GFP. $\mathrm{n}=8$. (C) Assessment of the superoxide anion level in lung adenocarcinoma cells. ${ }^{*} \mathrm{P}<0.05$ vs. Ad-GFP. $\mathrm{n}=8$.

Furthermore, Sirt3 overexpression downregulated ROS and superoxide anion levels in A549 lung adenocarcinoma cells. These data clearly suggest that Sirt3 is a tumor suppressor through the induction of apoptosis in lung adenocarcinoma cells.

The first important finding of our study is that Sirt3 was downregulated in human lung adenocarcinoma tissue. Acetylation was found in $>20 \%$ of mitochondrial proteins, including many longevity regulators and metabolism enzymes in a large-scale proteomics analysis (26), implicating the wide influence of acetylation modification among mitochondrial proteins. As acetylation modification is mainly mediated by sirtuins and Sirt3 is the major deacetylase of sirtuins in mitochondria (17), Sirt3 is believed to be a potent regulator in mitochondria. Furthermore, the energy status dictates the status of mitochondrial protein acetylation, suggesting that Sirt3-mediated mitochondrial acetylation may be a critical regulatory mechanism underlying the adaptive response to energy stress. In line with the potential role of Sirt3 in mitochondrial biology, metabolically active tissues with high oxidative capacity, such as skeletal muscle, liver, brain, kidney and adipose tissue, express Sirt3 abundantly (27). The downregulation of Sirt3 in lung adenocarcinoma may lead to enhanced acetylation status and ROS generation in mitochondria, which are tightly associated with higher cancer risk $(28,29)$.

Previously, significant reduction in the Sirt3 copy number was found in human breast cancer (30) and hepatocellular carcinoma $(21,31)$. Moreover, loss of Sirt3 in vitro led to tumorigenesis (19), and Sirt3 levels were decreased in human breast cancer $(19,20)$. In breast cancer cells, Finley et al (20) demonstrated that SIRT3 mediates metabolic reprogramming by destabilizing hypoxia inducible factor $1 \alpha$ (HIF-1 $\alpha$ ), a transcription factor that controls glycolytic gene expression.
Murine tumors lacking Sirt3 exhibit abnormally high levels of ROS that directly induce genomic instability and cellular metabolic reprogramming (20). Bell et al (32) also showed that Sirt3 suppressed tumor growth via inhibition of HIF-1 $\alpha$. All these data suggest the inhibitory effects of Sirt3 in tumor. However, we also noted a contradictory finding. Sirt 3 was upregulated in oral cancer and was required to protect oral cancer from stress-mediated cell death by various stimuli (33). In our studies, we found that Sirt3 was downregulated in human lung adenocarcinoma tissue, supporting the tumor-suppressive role of Sirt3 in lung adenocarcinoma. Subsequent functional analysis also showed that Sirt3 overexpression inhibited A549 lung adenocarcinoma cell proliferation.

The inhibitory effect of Sirt3 overexpression on lung adenocarcinoma cell growth was very obvious in vitro, which prompted us to study apoptosis. Using Annexin V + PI assay and cleaved caspase- 3 immunoblotting, we confirmed the apoptosis-inducing effects of Sirt3 in A549 lung adenocarcinoma cells. After revealing the pro-apoptotic feature of Sirt3, we observed that Sirt3 overexpression was modulated by apoptotic signaling pathways (bax, bcl-2, bad, bcl-x/L and AIF). Bcl-2 family proteins are known to play a pivotal role in the induction of mitochondrial caspase activation and in the regulation of apoptosis (34). AIF is a flavoprotein that is normally confined to the mitochondrial intermembrane space, yet translocates to the nucleus to induce peripheral chromatin condensation and triggers large-scale DNA degradation to fragments of $\sim 50 \mathrm{kbp}$ (35). In our study, overexpression of Sirt 3 increased bax-bcl- 2 and $\mathrm{bad} / \mathrm{bcl}-\mathrm{x} / \mathrm{L}$ ratios and induced AIF nuclear translocation. We postulated that these changes may contribute to the apoptotic-inducing effects of Sirt3 in A549 lung adenocarcinoma cells.

Finally, we provide evidence showing that Sirt3 overexpression was associated with an upregulated p53 protein level and a decreased ROS level in lung adenocarcinoma cells. Sirt1, another member of the sirtuin family, has been found to be able to directly deacetylate p53 and increase p53 protein level $(36,37)$. Recently, a study demonstrated that Sirt3 also enhanced the p53 protein level in hepatocellular carcinoma cells (21). In agreement with these studies, we observed that Sirt3 overexpression upregulated p53 and its downstream factor p21 in lung adenocarcinoma cells. Giving that p53 is a well-known tumor suppressor (38), we hypothesized that p53 upregulation is quite likely to contribute to the tumorsuppressive effect of Sirt3 in lung adenocarcinoma cells.

Collectively, we demonstrated that the expression of Sirt3 was decreased in human lung adenocarcinoma tissue. Overexpression of Sirt3 exhibited an obvious antitumor effect in the A549 lung adenocarcinoma cell line through induction of apoptosis. Our finding concerning the regulation of lung adenocarcinoma cell growth by Sirt3 may provide an important focus for the further understanding of lung adenocarcinoma and novel therapeutic interventions.

\section{Acknowledgements}

This study was supported by grants from the 'New Teachers Fund for Doctor Stations', Ministry of Education of China (no. 20100162120059) and the Fundamental Research Funds for the Central Universities (no. 2011QNZT175). 


\section{References}

1. Herbst RS, Heymach JV and Lippman SM: Lung cancer. N Engl J Med 359: 1367-1380, 2008.

2. Jemal A, Simard EP, Dorell C, et al: Annual report to the nation on the status of cancer, 1975-2009, featuring the burden and trends in human papillomavirus (HPV)-associated cancers and HPV vaccination coverage levels. J Natl Cancer Inst 105 175-201, 2013.

3. Gazdar AF: The molecular and cellular basis of human lung cancer. Anticancer Res 14: 261-267, 1994.

4. Graziano SL, Gamble GP, Newman NB, et al: Prognostic significance of K-ras codon 12 mutations in patients with resected stage I and II non-small-cell lung cancer. J Clin Oncol 17: 668-675, 1999.

5. Ding L, Getz G, Wheeler DA, et al: Somatic mutations affect key pathways in lung adenocarcinoma. Nature 455: 1069-1075, 2008.

6. Weir BA, Woo MS, Getz G, et al: Characterizing the cancer genome in lung adenocarcinoma. Nature 450: 893-898, 2007.

7. Molina JR, Yang P, Cassivi SD, Schild SE and Adjei AA: Non-small cell lung cancer: epidemiology, risk factors, treatment, and survivorship. Mayo Clin Proc 83: 584-594, 2008.

8. Mitsudomi T and Yatabe Y: Epidermal growth factor receptor in relation to tumor development: EGFR gene and cancer. FEBS J 277: 301-308, 2010.

9. DeBerardinis RJ, Lum JJ, Hatzivassiliou G and Thompson CB The biology of cancer: metabolic reprogramming fuels cell growth and proliferation. Cell Metab 7: 11-20, 2008

10. Jones RG and Thompson CB: Tumor suppressors and cell metabolism: a recipe for cancer growth. Genes Dev 23: 537-548, 2009.

11. Hanahan D and Weinberg RA: Hallmarks of cancer: the next generation. Cell 144: 646-674, 2011.

12. Kroemer G, Galluzzi L and Brenner C: Mitochondrial membrane permeabilization in cell death. Physiol Rev 87: 99-163, 2007.

13. Fulda S, Galluzzi L and Kroemer G: Targeting mitochondria for cancer therapy. Nat Rev Drug Discov 9: 447-464, 2010.

14. Mathupala SP, Ko YH and Pedersen PL: The pivotal roles of mitochondria in cancer: Warburg and beyond and encouraging prospects for effective therapies. Biochim Biophys Acta 1797: 1225-1230, 2010.

15. Haigis MC and Guarente LP: Mammalian sirtuins - emerging roles in physiology, aging, and calorie restriction. Genes Dev 20 2913-2921, 2006.

16. Milne JC and Denu JM: The Sirtuin family: therapeutic targets to treat diseases of aging. Curr Opin Chem Biol 12: 11-17, 2008

17. Lombard DB, Alt FW, Cheng HL, et al: Mammalian Sir2 homolog SIRT3 regulates global mitochondrial lysine acetylation. Mol Cell Biol 27: 8807-8814, 2007.

18. Ashraf N, Zino S, Macintyre A, et al: Altered sirtuin expression is associated with node-positive breast cancer. Br J Cancer 95: 1056-1061, 2006.

19. Kim HS, Patel K, Muldoon-Jacobs K, et al: SIRT3 is a mitochondria-localized tumor suppressor required for maintenance of mitochondrial integrity and metabolism during stress. Cancer Cell 17: 41-52, 2010.

20. Finley LW, Carracedo A, Lee J, et al: SIRT3 opposes reprogramming of cancer cell metabolism through HIFl $\alpha$ destabilization. Cancer Cell 19: 416-428, 2011.
21. Zhang YY and Zhou LM: Sirt3 inhibits hepatocellular carcinoma cell growth through reducing Mdm2-mediated p53 degradation. Biochem Biophys Res Commun 423: 26-31, 2012.

22. Wang P, Xu TY, Guan YF, Su DF, Fan GR and Miao CY: Perivascular adipose tissue-derived visfatin is a vascular smooth muscle cell growth factor: role of nicotinamide mononucleotide. Cardiovasc Res 81: 370-380, 2009.

23. Wang P, Xu TY, Guan YF, et al: Nicotinamide phosphoribosyltransferase protects against ischemic stroke through SIRT1-dependent adenosine monophosphate-activated kinase pathway. Ann Neurol 69: 360-374, 2011.

24. Wang P, Zhang RY, Song J, et al: Loss of AMP-activated protein kinase- $\alpha 2$ impairs the insulin-sensitizing effect of calorie restriction in skeletal muscle. Diabetes 61: 1051-1061, 2012.

25. Wang P, Guan YF, Du H, Zhai QW, Su DF and Miao CY: Induction of autophagy contributes to the neuroprotection of nicotinamide phosphoribosyltransferase in cerebral ischemia. Autophagy 8: 77-87, 2012.

26. Kim SC, Sprung R, Chen Y, et al: Substrate and functional diversity of lysine acetylation revealed by a proteomics survey. Mol Cell 23: 607-618, 2006.

27. Shi T, Wang F, Stieren E and Tong Q: SIRT3, a mitochondrial sirtuin deacetylase, regulates mitochondrial function and thermogenesis in brown adipocytes. J Biol Chem 280: 13560-13567, 2005.

28. Arif M, Senapati P, Shandilya J and Kundu TK: Protein lysine acetylation in cellular function and its role in cancer manifestation. Biochim Biophys Acta 1799: 702-716, 2010.

29. Benhar M, Engelberg D and Levitzki A: ROS, stress-activated kinases and stress signaling in cancer. EMBO Rep 3: 420-425, 2002.

30. Chin SF, Teschendorff AE, Marioni JC, et al: High-resolution aCGH and expression profiling identifies a novel genomic subtype of ER negative breast cancer. Genome Biol 8: R215, 2007.

31. Zhang CZ, Liu L, Cai M, et al: Low SIRT3 expression correlates with poor differentiation and unfavorable prognosis in primary hepatocellular carcinoma. PLoS One 7: e51703, 2012.

32. Bell EL, Emerling BM, Ricoult SJ and Guarente L: SirT3 suppresses hypoxia inducible factor $1 \alpha$ and tumor growth by inhibiting mitochondrial ROS production. Oncogene 30: 2986-2996, 2011

33. Alhazzazi TY, Kamarajan P, Joo N, et al: Sirtuin-3 (SIRT3), a novel potential therapeutic target for oral cancer. Cancer 117: 1670-1678, 2011.

34. Youle RJ and Strasser A: The BCL-2 protein family: opposing activities that mediate cell death. Nat Rev Mol Cell Biol 9: 47-59, 2008.

35. Susin SA, Lorenzo HK, Zamzami N, et al: Molecular characterization of mitochondrial apoptosis-inducing factor. Nature 397: 441-446, 1999.

36. Langley E, Pearson M, Faretta M, et al: Human SIR2 deacetylates p53 and antagonizes PML/p53-induced cellular senescence. EMBO J 21: 2383-2396, 2002.

37. Cheng HL, Mostoslavsky R, Saito S, et al: Developmental defects and p53 hyperacetylation in Sir2 homolog (SIRT1)-deficient mice. Proc Natl Acad Sci USA 100: 10794-10799, 2003.

38. Greenblatt MS, Bennett WP, Hollstein $M$ and Harris CC: Mutations in the p53 tumor suppressor gene: clues to cancer etiology and molecular pathogenesis. Cancer Res 54: 4855-4878, 1994. 